\title{
Resultados de un sistema de atención privado basado en un modelo de salud familiar en Chile
}

\author{
Álvaro Téllez $\mathrm{T}^{1}$, Elisa Aguayo $\mathrm{T}^{2 \mathrm{a}}$. \\ Results of a private primary health \\ system based on a family health care \\ model in Chile
}

Background: The health care network of a University in Chile started a family based primary health care system using pre paid model as financing. Aim: To describe the results of this family Health Care Center. Material and Methods: Between 1999 and 2006, the Pontificia Universidad Catolica-Health Network offered a family care plan characterized by a preferential access to a Family Care Center (CSF), that coordinated and solved all health care needs. Several indicators and perception of satisfaction were used to assess the results of this plan, in 2003 and 2004. Results: During the study period, 5,402 people were attended. Seventy three percent of these were aged between 15-44 years. The waiting lapse was less than 48 hours for medical appointments and less than 30 days for programmed surgical procedures. The coverage of preventive actions varied from $26 \%$ of mammographies among women between 25 to 69 years old to $100 \%$ for healthy child care among newborns. Thirty one percent of diabetics achieved a glycosilated hemoglobin of less than $7 \%$ and $19 \%$ of hypertensive subjects achieved blood pressure values below 135/85 mmHg. Family physicians had 2.3 appointments per person per year and solved $85 \%$ of the medical problems that motivated the consultation. In average, $88 \%$ of interviewed subjects evaluated the attention received as very good or excellent. Conclusions: The application of this model in the private sector achieves measurable health care results with a good degree of client satisfaction (Rev Méd Chile 2008; 136: 873-9).

(Key words: Delivery of health care; Hospitals, private; Managed care programs)

Recibido el 17 de julio, 2007. Aceptado el 13 de marzo, 2008.

${ }_{1}^{1}$ Departamento de Medicina Familiar, Facultad de Medicina, Pontificia Universidad Católica de Chile. ${ }^{2}$ Escuela de Enfermenía, Pontificia Universidad Católica de Chile. Santiago de Chile.

aEnfermera-Matrona

A comparar países que poseen sistemas de salud con fuerte desarrollo en atención primaria con países sin esta característica, se concluye que los primeros presentan un menor gasto en salud y logran mejores resultados expresados en: menor prevalencia de bajo peso de nacimiento,

Correspondencia a: Dr. Álvaro Téllez T. Departamento de Medicina Familiar, Facultad de Medicina, PUC. Lira 44, Santiago. Teléfono: 3546854. Fax: 3548122. E mail: atellez@med.puc.cl menor mortalidad infantil, menos años de vida perdidos y mayor expectativa de vida ${ }^{1,2}$. La Organización Panamericana de la Salud, basada en estos argumentos, promueve el fortalecimiento de la atención primaria, caracterizándola como el ámbito de primer contacto que ofrece cobertura universal y acceso a servicios aceptables para la población, prestando atención integral, integrada y apropiada a lo largo del tiempo, con énfasis en prevención y promoción en salud ${ }^{3}$. 
Chile ha sido uno de los países que ha desarrollado fuertemente la atención primaria en el sistema público de salud, lo que ha contribuido a la obtención de buenos resultados sanitarios, como por ejemplo, disminución en la mortalidad infantil producto de la reducción de la mortalidad por infecciones respiratorias bajas en menores de un año ${ }^{4}$. Sin embargo, el desarrollo de la atención primaria en el sistema de salud privado en Chile ha sido insuficiente; en este ámbito ha predominado más bien un enfoque curativo y una oferta asistencial basada en la competencia de atenciones especializadas, bajo el estímulo del sistema de financiamiento "pago por servicios".

La Red de Salud de la Pontificia Universidad Católica de Chile (PUC), una de las organizaciones de salud privada más grande del país, decidió en 1999 ofrecer un sistema de atención de amplia cobertura, basado en el modelo de salud familiar, coordinado desde un centro de atención primaria y financiado por un modelo de prepago per cápita. El objetivo de este artículo es caracterizar a la población atendida bajo el modelo de salud familiar y describir los principales resultados clínicos y de satisfacción en la población expuesta a este sistema.

\section{MATERIAL y MÉTOdO}

Entre octubre de 1999 y diciembre de 2006 se ofreció a la población un Plan de Salud Familiar (PSF), comercializado a través de dos Instituciones de Salud Previsional (ISAPRE). Las personas que se afiliaban a este plan de salud adquirían el derecho a una atención preferencial en la Red de Salud de la PUC, con una amplia cobertura ambulatoria y hospitalaria, con libre acceso al servicio de urgencia y a la atención primaria, que otorgaba el Centro de Salud Familiar San Joaquín (CSF). El acceso a las atenciones especializadas, hospitalización y a toda la gama de servicios clínicos de apoyo, era coordinado por el CSF. Un porcentaje del valor de la cotización de salud recaudado por la ISAPRE, por cada persona afiliada a este plan de salud, era transferido a la Red de Salud PUC mensualmente, lo que sumado a copagos fijos, debían financiar el costo de los servicios asistenciales, incluyendo los subsidios por incapacidad laboral. El CSF se comprometía a prestar la mayoría de las atenciones que la población requería, bajo el marco del modelo de atención en salud familiar ${ }^{5}$. Los principales componentes de este modelo fueron:

- Equipo de salud de cabecera: cada persona y familia era atendida en forma preferencial por un mismo equipo de profesionales, con quien establecía una relación continua y resolvía sus problemas en salud.

- Enfoque familiar: el equipo de salud aplicó un enfoque biosicosocial y sistémico, incorporando el contexto familiar y social como un referente para el diagnóstico y la intervención.

- Atención integral: se ofreció un amplio espectro de atención: curativa, preventiva y promocional, lo que significó organizar un plan programado de prestaciones.

- Autocuidado. Se promovió en las personas un rol más activo y responsable en el cuidado de su salud y en la toma de decisiones.

Para ofrecer este modelo de atención se seleccionó un equipo de salud formado por: médicos familiares con mención niño y mención adulto, médicos obstetras, enfermeras-matronas, auxiliar de enfermería y administrativos.

El CSF ofreció acceso de atención durante $10 \mathrm{~h}$ diarias ( $8: 30 \mathrm{~h}$ a 18:30 h) de lunes a viernes. Fuera de este horario, la población debía recurrir al Servicio de Urgencia de la Red de Salud PUC.

Se incorporaron como elementos de apoyo al modelo de atención, un registro clínico electrónico (OMI-AP Inet versión 6.2/00A-02 España), guías de práctica clínica basadas en evidencias adaptadas por el equipo de salud, un sistema de atención telefónica y un sistema de monitoreo de la calidad incorporado en el Programa de Mejoramiento Continuo de la Red de Salud PUC.

El presente estudio, de tipo observacional descriptivo, se realizó sobre toda la población afiliada al CSF en el período comprendido entre el 1 de enero de 2003 y el 31 de diciembre de 2004. Se seleccionaron indicadores que ilustran calidad asistencial en organizaciones sanitarias ${ }^{6,7}$, susceptibles de ser construidos con la información disponible en el registro clínico electrónico y en el sistema de información de la Red de Salud PUC. Específicamente se midió: oferta asistencial, tiempos de espera, coberturas y capacidad resolutiva, expresada como el porcentaje de consultas resuel- 
tas y la distribución de las derivaciones según especialidades. Además se midieron los recursos asistenciales utilizados por la población como: tasas de consultas, de exámenes de laboratorio y de imágenes. Finalmente, se midió la satisfacción de los usuarios mediante la aplicación de una encuesta de opinión a una muestra representativa, aleatoria y estratificada de pacientes en espera en el CSF en diciembre de 2003. El tamaño de esta muestra se calculó considerando un error de 5\% para un nivel de confianza de 95\%. Este instrumento fue aplicado por entrevistadores externos entrenados, a 64 pacientes mayores de 15 años. La encuesta de opinión indagó las dimensiones de estructura, proceso y resultado de la atención, a través de preguntas cerradas con respuestas clasificadas según categorías de Likert.

\section{Resultados}

Durante el período 2003-2004 se mantuvieron afiliadas al PSF 5.402 personas y $83 \%$ de ellas tuvo contacto con el CSF. La permanencia promedio en el plan fue de 22 meses, con un rango entre $17 \mathrm{y}$ 38 meses. El 73\% de la población estaba en el rango etáreo de los 15-44 años, con un predominio masculino (70\%) (Tabla 1). El segmento socioeconómico de la población afiliada al PSF pertenecía a la categoría C3 y D (nivel medio) ${ }^{8}$ con un ingreso familiar promedio entre $\$ 150.000 \mathrm{y}$ $\$ 350.000$ mensual.

El CSF dispuso de un médico de cabecera por cada 2.500 personas y de una jornada enfermera por cada 2.770 personas. La espera máxima por una atención médica no urgente en el CSF fue de 2 días, para una consulta de especialidad fue de 14 días y para una cirugía programada fue de un mes.

Las coberturas logradas en el CSF en prestaciones preventivas relacionadas con la salud de la mujer, del niño y del adulto se muestran en la Tabla 2, las que fluctuaron entre $26 \%$ de cobertura en mamografías para las mujeres entre 40 y 69 años y $100 \%$ de cobertura en la supervisión del recién nacido. Otros indicadores relevantes para la salud de la población indicaron que: la resolu-

Tabla 1. D istribución de la población afiliada al PSF según edad y sexo. Período 2003-2004

\begin{tabular}{|lccr|}
\hline Grupo etáreo & Masculino & Femenino & Total \\
$\mathrm{n}=3.761(70 \%)$ & $\mathrm{n}=1.640(30 \%)$ & $\mathrm{n}=5.402$ \\
\hline 0 a 14 años & $499(13 \%)$ & $490(30 \%)$ & 988 \\
15 a 29 años & $2.222(59 \%)$ & $541(33 \%)$ & 2.763 \\
30 a 44 años & $802(21 \%)$ & $391(24 \%)$ & 1.193 \\
45 a 64 años & $236(6 \%)$ & $212(13 \%)$ & 448 \\
65 y más años & $3(0,1 \%)$ & $6(0,4 \%)$ & 9 \\
\hline
\end{tabular}

Tabla 2. Coberturas de prestaciones preventivas en la población afiliada al PSF. Período 2003-2004

\begin{tabular}{|lc|}
\hline Prestación & Población atendida/población objetivo \\
\hline Control embarazo antes de las 14 semanas de gestación & $73 \%$ \\
Ultrasonografía antes de las 20 semanas de gestación & $73 \%$ \\
Consulta de puerperio antes de los 15 días & $88 \%$ \\
PAP en mujeres 25-44 años & $62 \%$ \\
Mamografía en mujeres 40-69 años & $26 \%$ \\
Supervisión del recién nacido & $100 \%$ \\
Supervisión del lactante entre 1 y 23 meses & $77 \%$ \\
Examen preventivo 20-64 años & $55 \%$ \\
Control de personas con HTA >15 años & $49 \%$ \\
Control de personas con diabetes mellitus II >15 años & $32 \%$ \\
\hline
\end{tabular}


ción de los partos se hizo en 78,8\% por vía vaginal; $57 \%$ de los niños de seis meses se alimentaron con lactancia materna exclusiva; el porcentaje de personas portadoras de una diabetes mellitus II que tenía hemoglobina glicosilada (HbA1c) menor a 7 alcanzó en este período a $31 \%$, mientras que en el caso de la hipertensión arterial, el 19\% tuvo presiones arteriales menores a $135 \mathrm{mmHg}$ de sistólica y a $85 \mathrm{mmHg}$ de diastólica.

El número total de consultas atendidas en el CSF en el período 2003-2004 fue de 14.013; de éstas, $85 \%$ fue resuelta por el médico de cabecera y el $15 \%$ restante fue derivada a especialistas, principalmente a oftalmología, traumatología, dermatología y otorrinolaringología (Tabla 3). Por cada 100 consultas se realizaron 53 exámenes de laboratorio y 18 exámenes de imagen. La Tabla 4 muestra las tasas de diferentes prestaciones, incluidas en el PSF a las que se debe agregar la indicación de 4,7 días de incapacidad laboral por trabajador afiliado al PSF el año 2004, con un promedio de 6,9 días por licencia médica.

La calidad pencibida por los afiliados al PSF, medida mediante la encuesta de opinión, fue calificada en promedio como excelente o muy buena por $88 \%$ de los encuestados. El 100\% de los encuestados calificó de excelente o muy buena la atención por la enfermera de cabecera, la atención en el servicio de urgencia y en el hospital. La dimensión de más baja evaluación fue el horario del CSF, en que 59\% de los encuestados lo calificó como excelente o muy bueno (Tabla 5).

\section{DisCUSIÓN}

El plan de atención descrito en este reporte constituye una de las primeras experiencias informadas de un sistema de atención primaria basado en un modelo de salud familiar, al interior de una red privada de atención en Chile. El hecho de

\section{Tabla 3. D istribución de las derivaciones realizadas en el C SF según las especialidades de destino (período 2003-2004)}

\begin{tabular}{|lrr|}
\hline Especialidad & $\mathrm{N}^{\circ}$ & $\%$ \\
\hline Oftalmología & 480 & 26 \\
Traumatología & 310 & 17 \\
Psiquiatría & 278 & 15 \\
Dermatología & 214 & 12 \\
Otorrinolaringología & 196 & 11 \\
Cirugía & 83 & 4 \\
Neurología & 80 & 4 \\
Urología & 58 & 3 \\
Oncología & 37 & 2 \\
Endocrinología & 22 & 1 \\
Gastroenterología & 19 & 1 \\
Reumatología & 15 & 1 \\
Nefrología & 9 & 0 \\
Cardiología & 8 & 0 \\
Bronco-pulmonar & 5 & 0 \\
Otras & 38 & 2 \\
Total & 1.852 & 100 \\
\hline
\end{tabular}

Tabla 4. Tasas de prestaciones* en la población afiliada al PSF. Período 2003-2004

\begin{tabular}{|lcr|}
\hline Prestaciones & 2003 & 2004 \\
Consultas medicina familiar & 1,64 & 1,39 \\
Consulta de especialidades & 0,52 & 0,55 \\
Consultas de urgencia & 0,21 & 0,27 \\
& & \\
Total consultas médicas & 2,37 & 2,21 \\
Exámenes de laboratorio & 1,76 & 1,65 \\
Exámenes de rayos & 0,41 & 0,50 \\
Quinesioterapia & 0,01 & 0,10 \\
Procedimientos & 0,22 & 0,89 \\
Hospitalizaciones & 0,059 & 0,056 \\
\hline
\end{tabular}

*Número de prestaciones por persona al año 
Tabla 5. Evaluación calidad percibida por pacientes del PSF ( $n=64)$. D iciembre 2003

\begin{tabular}{|lccrcc|}
\hline Criterio & Excelente & Muy buena & Buena & Regular & Malo \\
\hline Atención por médico de cabecera en el CSF & $63 \%$ & $32 \%$ & $5 \%$ & - & - \\
Atención por enfermera de cabecera en el CSF & $63 \%$ & $37 \%$ & - & - & - \\
Atención en el hospital PUC & $62 \%$ & $38 \%$ & - & - & - \\
Atención en la urgencia PUC & $63 \%$ & $37 \%$ & - & - & - \\
Respuesta a los reclamos & $39 \%$ & $41 \%$ & $10 \%$ & $5 \%$ & $5 \%$ \\
Resolución del problema de salud en el CSF & $42 \%$ & $46 \%$ & $7 \%$ & $4 \%$ & $2 \%$ \\
Trato en el CSF & $60 \%$ & $35 \%$ & $3 \%$ & $2 \%$ & \\
Horario de atención del CSF & $23 \%$ & $36 \%$ & $22 \%$ & $14 \%$ & $5 \%$ \\
Comunicación telefónica con el CSF & $40 \%$ & $38 \%$ & $16 \%$ & $5 \%$ & $1 \%$ \\
Accesibilidad al CSF & $42 \%$ & $33 \%$ & $17 \%$ & $6 \%$ & $2 \%$ \\
Proceso de recepción en el CSF & $59 \%$ & $31 \%$ & $10 \%$ & - & - \\
Satisfacción global con la atención & $43 \%$ & $54 \%$ & $3 \%$ & - & - \\
\hline
\end{tabular}

poder atender a una población definida y estable permitió medir resultados que normalmente no están disponibles en el sistema privado, a diferencia de lo que ocurre en el sistema de salud público, donde estos indicadores son reportados en forma habitual. El PSF logró un buen nivel de coberturas (rango 26\%-100\%) en la mayoría de las prestaciones preventivas, un alto porcentaje de resolución de las consultas del médico de cabecera (85\%), buenos desempeños en otros indicadores de calidad asistencial (78,8\% de partos por vía vaginal, $57 \%$ de niños de 6 meses con lactancia materna exclusiva; $31 \%$ de pacientes con diabetes tipo II compensados) y un alto grado (88\%) de satisfacción entre sus usuarios.

La metodología de este estudio no permite hacer una comparación de esta experiencia con otras provenientes del sector privado o público, porque el perfil demográfico de la población expuesta a este plan de atención no es representativo de la población general, ni tampoco de la población adscrita al sector ISAPRE. Por estos motivos, no es posible concluir si el PSF tuvo un desempeño mejor que el del resto del sistema de atención.

No es mucha la información disponible que permite comparar los sistemas de salud público y privado en Chile. En cuanto a oferta de atención médica, es indudable que este recurso es más abundante en el sistema privado, que otorgó 4,1 consultas por persona/año en $2004^{9}$, mientras que el sistema público ofreció, incluidas las atenciones institucionales y las de libre elección del Fondo Nacional de Salud, un promedio de 1,51 consultas por persona ese mismo año ${ }^{10,11}$. El resultado del PSF, de 2,21 consultas por persona/año, lo ubica más cerca de la oferta producida por el sistema público.

Cabe destacar que en el PSF, donde el médico de cabecera podía indicar derivaciones sin restricciones, sólo $15 \%$ de las consultas realizadas en el CSF fueron referidas a especialistas.

En el sistema de atención privada no hay a la fecha información disponible sobre coberturas de prestaciones preventivas, con excepción del reporte de $31 \%$ de cobertura de Papanicolau ${ }^{12}$, cifra inferior a $62 \%$ logrado por el PSF. Con relación a la compensación en enfermedades crónicas, la cifra obtenida por el PSF de 31\% para diabetes fue superior a $25 \%$ reportado por la Encuesta Nacional de Salud del año 200313, sin embargo $19 \%$ de compensación obtenido en hipertensión arterial, fue inferior a 33\% reportado en la misma encuesta.

Por otra parte, destacamos algunos indicadores relacionados con el uso de los recursos asistenciales, que pueden estar relacionados con el incentivo que establece el sistema de financiamiento de prepago per cápita. Por ejemplo, la proporción de partos resueltos por cesárea en el PSF $(21,2 \%)$ fue inferior a $61,1 \%$ informado para el sistema privado el año $2004^{14}$, pero algo superior al estándar recomendado por la OMS que es de $15 \%{ }^{15}$. Por 
otra parte, el índice de 54 exámenes de laboratorio por cada 100 consultas del PSF es prácticamente la mitad que el calculado para el sector ISAPRES que llega a 119 exámenes/100 consultas ${ }^{9}$; asimismo, el promedio de 6,9 días por licencia médica en el PSF fue menor al 9,1 informado por las ISAPRES en $2004^{16}$.

En lo relacionado con la satisfacción, 88,1\% de los encuestados calificó como muy bueno o excelente los diferentes aspectos del sistema de atención del PSF. El referente más cercano para contextualizar estos resultados es el Estudio Nacional sobre Satisfacción y Gasto en Salud realizado por el Ministerio de Salud en $2005^{17}$, que mostró 93,7\% de satisfacción global en atención ambulatoria en prestadores privados, cifra inferior a $97 \%$ alcanzado por el PSF en este criterio.

Los resultados de esta experiencia, en nuestro conocimiento la primera reportada de un sistema privado de salud, demuestran que un modelo de atención preocupado no sólo de responder a la demanda por atención médica, sino de actuar también con intención preventiva, permite obtener resultados de salud cuantificables y comparables con los del sector público. Asimismo, muestra un sistema de financiamiento de prepago que estimula el uso eficiente de los recursos. Sin embargo, a pesar de los atributos descritos, este modelo no logra resultados satisfactorios en un indicador importante, como el de compensación de hipertensión arterial. Desgraciadamente, no sería extraño que el resto del sistema de atención privado en Chile, tenga cifras incluso más bajas en el control de enfermos crónicos, pues en general éste carece de incentivos dirigidos hacia la pre-

\section{REFERENCIAS}

1. StaRfield B. Is primary care essential? Lancet 1994; 344: 1129-33.

2. Macinko J, Starfield B, Shi L. The contribution of primary care systems to health outcomes within OECD countries, 1970-1998. Health Services Research 2003; 38: 819-53.

3. OPS. Renovación de la Atención Primaria de Salud en las Américas. Documento de Posición 2005. Disponible en: http://www.paho.org/spanish/ad/ vención clínica y hacia la obtención de resultados en la salud de su población.

Más allá del presente reporte, quisiéramos resaltar la expeniencia del PSF como un modelo de fortalecimiento de la atención primaria en el sector privado, adjudicándole a ésta un rol coordinador del resto de la red de atención. Esto sigue la línea de las recomendaciones de la OMS/OPS 3 , que a su vez están basadas en evidencias y en experiencias que han logrado demostrar las ventajas de esta estrategia. Una de las más claras es la expeniencia de Kaiser Permanente en Estados Unidos de Norteamérica ${ }^{18}$, que es un buen ejemplo de cómo una entidad de salud privada, orientada a mejorar la salud de su población, poniendo énfasis en el acceso a la atención primania y en la coordinación de toda la red de atención, no sólo logra buenos resultados sanitarios sino también una buena eficiencia en el uso de los recursos.

En conclusión, la experiencia del PSF ha demostrado que es posible ofrecer un sistema de atención basado en la atención primaria en el sector privado y que cuando éste opera en condiciones regulares, permite obtener resultados de salud cuantificables en la población atendida y susceptible de ser comparados con los del resto del sistema de atención. La población afiliada mostró altos niveles de satisfacción con esta modalidad de atención.

\section{Agradecimientos}

Al Dr. José A. Castro-Rodríguez por su colaboración en la revisión de este artículo.

A todo el equipo del Centro de Salud Familiar San Joaquín, especialmente a su Directora, la Dra. Verónica Rojas, que permitieron la realización de este estudio.

ths/os/phc2ppaper_10-ago-05_spa.pdf. Consultado el 23 de mayo $200 \overline{7}$.

4. JimÉNEZ J, Romero MI. Reducing Infant Mortality in Chile: Success in Two Phases. Health Affaire 2007; 26: 458-65.

5. Rojas P, Télez A. Modelo Biosicosocial. En: Montero J. Medicina Ambulatoria del Adulto. Santiago: Ediciones Universidad Católica de Chile, 2001; 344-9.

6. Peiró S, CASAS M. Comparación de la actividad y resultados de los hospitales en España y perspectivas. En: Cabasés JM, Villalbí JR, Aibar C. Invertir 
para la salud. Prioridades en salud pública. Informe Sespas 2002. España: Editorial Artes Gráficas Soler, SL; Pgs. 511-28.

7. Ministerio de Sanidad y Consumo de España. Calidad asistencial en la Unión Europea. Cap. 3 Informe anual año 2005. Disponible en: www.msc.es/organizacion/sns/informeAnualSNS/ docs/2005/. Consultado el 15 de mayo 2007.

8. MÉNDEZ R. Segmentación socioeconómica. Disponible en: http://www.monografias.com/trabajos6/seme/ seme.shtml. Consultado el 23 de mayo 2007.

9. Superintendencia de Salud Departamento de Estudio y Desarrollo. Perfil de Prestaciones Médica en el Sistema Isapre 2004 Disponible en: http:// www.supersalud.cl/d ocumentacion/569/article2468.html. Consultado el 23 de mayo 2007.

10. MINSAL, Atención médica por servicio de salud, nivel de atención y programas. SNSS, 2004. Disponible en: http://deis.minsal.cl/deis/codigo/neuw/ at medicas 2004.asp. Consultado el 6 de junio 2007.

11. FONASA Boletín estadístico 2003-2004 Disponible en: https://fon.fonasa.cl/prontus fonasa/site/artic/ 20070112/asocfile/boletin estadistico 20032004_version_final_pdf_25_07_06_pdf. Consultado el 11 de junio 2007.

12. Superintendencia de Salud Departamento de Estudio y Desarrollo Documento de Trabajo Examen de
Medicina Preventiva durante el Primer Año de Vigencia 2005. Disponible en: http://www.supersalud.cl/ documento/560/articles-1069_recurso_1.9DF. Consultado el 23 de mayo 2007.

13. MINSAL Encuesta Nacional de Salud 2003. Disponible en: http://epi.minsal.cl/epi/html/invest/ENS/ ENS.htm. Consultado el 20 marzo 2007.

14. Superintendencia de Salud Departamento de Estudio y Desarrollo Perfil de Prestaciones Médicas en el Sistema Isapre 2004. Disponible en: www.supersalu.cl/documentacion/569/article-2468.html. Consultado el 23 de mayo 2007.

15. OMS. Appropriate technology for birth. Lancet 1985; 2: 436-7.

16. Superintendencia de Salud Departamento de Estudio y Desarrollo. Estadísticas de Licencias Médicas Año 2004 Disponible en: www.supersalud.cl/documentacion/569/article-2185.html. Consultado el 13 de junio 2007.

17. MINSAL Objetivos sanitarios para la década 20002010. Evaluación a mitad de período. Disponible en: epi.minsal.cl/epi/html/elvigia/vigia15.pdf. Consultado el 30 de abril 2007.

18. Feachem RGA, SeKhri NK, White KL. Getting more for their dollar: a comparison of the NHS with California's Kaiser Permanente. BMJ 2002; 324: 135-43. 